\title{
Lyapunov-Based Controller for a Class of Stochastic Chaotic Systems
}

\author{
Hossein Shokouhi-Nejad, Amir Rikhtehgar Ghiasi, and Saeed Pezeshki \\ Faculty of Electrical and Computer Engineering, University of Tabriz, Tabriz 5166614776, Iran \\ Correspondence should be addressed to Hossein Shokouhi-Nejad; h.shokouhinejad@tabrizu.ac.ir
}

Received 25 July 2014; Accepted 4 November 2014; Published 10 December 2014

Academic Editor: Zhang Yi

Copyright (C) 2014 Hossein Shokouhi-Nejad et al. This is an open access article distributed under the Creative Commons Attribution License, which permits unrestricted use, distribution, and reproduction in any medium, provided the original work is properly cited.

\begin{abstract}
This study presents a general control law based on Lyapunov's direct method for a group of well-known stochastic chaotic systems. Since real chaotic systems have undesired random-like behaviors which have also been deteriorated by environmental noise, chaotic systems are modeled by exciting a deterministic chaotic system with a white noise obtained from derivative of Wiener process which eventually generates an Ito differential equation. Proposed controller not only can asymptotically stabilize these systems in meansquare sense against their undesired intrinsic properties, but also exhibits good transient response. Simulation results highlight effectiveness and feasibility of proposed controller in outperforming stochastic chaotic systems.
\end{abstract}

\section{Introduction}

In last two decades, the problem of control of chaotic systems has been widely investigated by many researchers due to the existence of chaos in real practical systems [1-10]. A chaotic system has some inherent characteristics, such as excessive sensitivity to initial conditions, fractal properties of the motion in phase space, and board spectrums of the frequency response; hence, it is usually difficult to accurately predict the future behavior of the chaotic system, which can end up in performance degradation and restriction on the operating range of dynamic systems.

Considering aforesaid, developing strategies for controlling chaos phenomenon based on the features of chaotic motion is highly important; therefore, many nonlinear techniques for chaos control were proposed, such as feedback control $[1,2]$ and sliding mode control [3-5]. To exploit their advantages, these approaches are integrated as a complex control algorithm such as adaptive sliding mode [6,7], adaptive fuzzy sliding mode control [8], and predictive feedback control [9]. However, in practice, real systems are usually affected by external perturbations which, in many cases, are of great uncertainty and hence may be treated as random; therefore, controlling chaos in such concrete systems needs to be regarded by stochastic concepts. Stochastic chaotic systems appear in many fields of science and engineering such as mechanical engineering [10], biology systems [11, 12], chemistry [13], physics and laser science [14], and financial systems [15]. For modeling stochastic chaotic systems, an Ito stochastic differential form is utilized by using the derivative of a Wiener process which creates a white Gaussian noise [16].

In [17], the sliding mode control is used for controlling stochastic chaos toward desired unstable periodic orbits of the deterministic chaotic system but the sliding mode suffers from the deficiency (chatter), which is caused by the sign function switch term in the control input. As a result, the convergence of the stochastic states to the desired equilibrium point cannot be completely achieved and state variance converges to a bound around equilibrium point. In other words, they cannot satisfy asymptotical stability condition for stochastic chaotic systems in mean-square sense.

According to the above, the main contribution of this paper is design of a very simple controller for a group of stochastic chaotic systems on the basis of Lyapunov's direct method which guarantees the asymptotically stabilizing of these systems in mean-square sense. Therefore, by adding stochastic terms to the generalized form of a well-known group of chaotic systems, control problem of these systems 
is investigated. The Lyapunov direct method which is a useful tool for designing globally stabilizing control schemes is utilized in this work. One of the important advantages of this method is stabilizing systems globally without any linearization. Numerical simulations show that the proposed method can easily eliminate undesired characteristics of chaos phenomenon and stabilize the system.

This paper is organized as follows: general description of a group of chaotic systems is presented in Section 2. In Section 3, Lyapunov-based control law is obtained and stability of the proposed scheme is analyzed. In Section 4, numerical simulation results are shown. Finally, conclusion is addressed in Section 5.

Notation. In this paper, $L_{2}[0, \infty)$ is the space of square-integrable vector function over $[0, \infty),\|\|$ stands for the usual $L_{2}[0, \infty)$ norm, and $\left(\Omega, \mathscr{F},\left\{\mathscr{F}_{t}\right\}_{t \in R}, \mathscr{P}\right)$ is a complete probability space with a filtration $\left\{\mathscr{F}_{t}\right\}_{t \in R}$ satisfying the usual conditions (i.e., filtration contains all $\mathrm{P}$-null sets and is right continuous) [18].

\section{System Description}

Consider a class of three-dimensional chaotic system described as [6]

$$
\begin{aligned}
{\left[\begin{array}{c}
\dot{x}(t) \\
\dot{y}(t) \\
\dot{z}(t)
\end{array}\right]=} & {\left[\begin{array}{ccc}
-\alpha & 0 & 0 \\
g(s(t)) & -\beta & \psi(s(t)) \\
h(s(t)) & -\psi(s(t)) & -\gamma
\end{array}\right]\left[\begin{array}{c}
x(t) \\
y(t) \\
z(t)
\end{array}\right] } \\
+ & {\left[\begin{array}{c}
f(s(t)) \\
0 \\
0
\end{array}\right], }
\end{aligned}
$$

where $x(t), y(t)$, and $z(t)$ are state variables and $\alpha, \beta, \gamma$ are nonnegative known constants. All of four functions $f(s(t))$, $g(s(t)), h(s(t))$, and $\psi(s(t))$ are considered as smooth functions, which belong to $\mathfrak{R}^{3} \rightarrow \mathfrak{R}$ space and $s(t)=(x(t)$, $y(t), z(t))$.

Remark 1. Note that, in [6], half of recent published chaotic systems are organized by (1). Adding financial system, Table 1 illustrates these chaotic models.

Taking the consideration of control input vector $u(t)$ and stochastic terms, the system can be expressed by

$$
\begin{aligned}
{\left[\begin{array}{c}
\dot{x}(t) \\
\dot{y}(t) \\
\dot{z}(t)
\end{array}\right]=} & {\left[\begin{array}{ccc}
-\alpha & 0 & 0 \\
g(s(t)) & -\beta & \psi(s(t)) \\
h(s(t)) & -\psi(s(t)) & -\gamma
\end{array}\right]\left[\begin{array}{l}
x(t) \\
y(t) \\
z(t)
\end{array}\right] } \\
& +\left[\begin{array}{c}
f(s(t)) \\
0 \\
0
\end{array}\right]+\left[\begin{array}{l}
u_{1}(t) \\
u_{2}(t) \\
u_{3}(t)
\end{array}\right]+\left[\begin{array}{l}
n_{1}(s(t), t) \dot{\omega}_{1} \\
n_{2}(s(t), t) \dot{\omega}_{2} \\
n_{3}(s(t), t) \dot{\omega}_{3}
\end{array}\right],
\end{aligned}
$$

where $n_{i}(s, t), \quad i=1,2,3: \mathfrak{R}^{n} \times \mathfrak{R}^{+} \rightarrow \mathfrak{R}$ is a nonlinear and sufficiently smooth function and $\omega_{i}, i=1,2,3$, are zeromean scalar Wiener process (Brownian motion) on $(\Omega, F, P)$ with a natural filtration $\left\{\mathscr{F}_{t}\right\}_{t \in R}$ and $\dot{\omega}_{i}=d \omega_{i} / d t$ are white Gaussian noise. Here, each white Gaussian noise $\omega_{i}$ is independent of other ones. Due to some technicalities and restrictions used in definition of $\dot{\omega}$ [16], system (2) has to be rewritten as follows:

$$
\begin{aligned}
& {\left[\begin{array}{l}
d x \\
d y \\
d z
\end{array}\right]} \\
& =\left(\left[\begin{array}{ccc}
-\alpha & 0 & 0 \\
g(s(t)) & -\beta & \psi(s(t)) \\
h(s(t)) & -\psi(s(t)) & -\gamma
\end{array}\right]\left[\begin{array}{l}
x(t) \\
y(t) \\
z(t)
\end{array}\right]\right. \\
& \left.+\left[\begin{array}{c}
f(s(t)) \\
0 \\
0
\end{array}\right]+\left[\begin{array}{l}
u_{1}(t) \\
u_{2}(t) \\
u_{3}(t)
\end{array}\right]\right) d t \\
& +\left[\begin{array}{l}
n_{1}(s(t), t) d \omega_{1} \\
n_{2}(s(t), t) d \omega_{2} \\
n_{3}(s(t), t) d \omega_{3}
\end{array}\right] .
\end{aligned}
$$

Assumption 2. The function $n_{i}(s, t)$ is assumed to be locally Lipschitz continuous and satisfies the linear growth condition as well and

$$
\|N(s(t), t)\| \leq\|G s(t)\|,
$$

where $N(s(t), t)=\left[n_{1}(s(t), t) \quad n_{2}(s(t), t) \quad n_{3}(s(t), t)\right]^{T}$ and $G_{3 \times 3}$ is a known matrix; therefore, the dynamics (3) possesses a unique global solution on $t \geq 0$, for any initial condition [19-21].

\section{Stochastic Chaos Control}

In this section, a nonlinear control law is proposed for a group of stochastic chaotic systems (3) based on Lyapunov's direct method. Lyapunov's direct method design is a systematic Lyapunov-based control technique, which can be applied to strict feedback systems, pure-feedback systems, and block strict-feedback systems. Prior to the move into design control law, two important definitions should be stated.

Definition 3 (see [22, 23]). The stochastic system (3) is said to be robustly stable in the mean-square sense if, for any scalar $\varepsilon>0$, there exists a scalar $\sigma(\varepsilon)>0$ such that $E\left\{\|x(t)\|^{2}\right\}<\varepsilon$, $E\left\{\|y(t)\|^{2}\right\}<\varepsilon$, and $E\left\{\|z(t)\|^{2}\right\}<\varepsilon$, for all $t>0$ for any initial conditions that support $\sup _{\tau \leq 0} E\left\{\left\|\phi_{x}(\tau)\right\|^{2}\right\}<\sigma(\varepsilon)$, $\sup _{\tau \leq 0} E\left\{\left\|\phi_{y}(\tau)\right\|^{2}\right\}<\sigma(\varepsilon)$, and $\sup _{\tau \leq 0} E\left\{\left\|\phi_{z}(\tau)\right\|^{2}\right\}<\sigma(\varepsilon)$. Additionally, system (3) is said to be asymptotically stable in the mean-square sense if $\lim _{t \rightarrow \infty}\left\{\|x(t)\|^{2}+\|y(t)\|^{2}+\right.$ $\left.\|z(t)\|^{2}\right\}=0$ holds for any initial condition.

Definition 4 (Itô formula [18]). Consider an $n$-dimensional stochastic vector process $\{X(t, \omega)\}$ with stochastic differential $d X(t, \omega)=F(t, \omega) \cdot d t+R(t, \omega) \cdot d W_{t}(\omega)$ on $t \geq 0$, where $W_{t}(\omega)$ is an $m$-dimensional Brownian motion defined on $\left(\Omega, \mathscr{F},\left\{\mathscr{F}_{t}\right\}_{t \in R}, \mathscr{P}\right)$. Let $V \in\left(\mathfrak{R}^{n} \times \mathfrak{R}^{+} ; \mathfrak{R}^{+}\right)$; then

$$
d V(t)=L V(t) d t+V_{x}^{T} R d \omega,
$$

where $L V(t)$ is given by

$$
L V(t)=V_{t}+V_{x}^{T} F+\frac{1}{2} \operatorname{tr}\left\{R R^{T} V_{x x}\right\},
$$


TABLE 1: List of published chaotic models, which belong to the general class.

\begin{tabular}{|c|c|c|c|c|c|c|}
\hline Number & Name & Model & $f(\cdot)$ & $g(\cdot)$ & $\psi(\cdot)$ & $\overline{h(\cdot)}$ \\
\hline 1 & Chen's system $(x(t) \rightleftarrows y(t))^{*}$ & $\left\{\begin{array}{l}\dot{x}=d y-y z+c x \\
\dot{y}=a(x-y) \\
\dot{z}=x y-b z\end{array}\right.$ & $d y-y z+c x$ & $a$ & 0 & $y$ \\
\hline 2 & Liu system & $\left\{\begin{array}{l}\dot{x}=a(y-x) \\
\dot{y}=b x-k x z \\
\dot{z}=-c z+h x^{2}\end{array}\right.$ & ay & $b-k z$ & $h x-z$ & 0 \\
\hline 3 & Lorenz model & $\left\{\begin{array}{l}\dot{x}=-a(x-y) \\
\dot{y}=r x-y-x z \\
\dot{z}=x y-b z\end{array}\right.$ & ay & $r-z$ & $y$ & 0 \\
\hline 4 & Lotka Volterra generalized-3D & $\left\{\begin{array}{l}\dot{x}=x-x y+c x^{2}-a z x^{2} \\
\dot{y}=r x-y-x z \\
\dot{z}=x y-b z\end{array}\right.$ & $-x y+c x^{2}-a z x^{2}$ & $r-z$ & $y$ & 0 \\
\hline 5 & Lu-Chen system $(x \rightleftarrows y)^{*}$ & $\left\{\begin{array}{l}\dot{x}=-x y+c x \\
\dot{y}=a(x-y) \\
\dot{z}=x y-b z\end{array}\right.$ & $-x y+c x$ & $a$ & $y$ & 0 \\
\hline 6 & Nose-Hoover system $(x \rightleftarrows y)^{*}$ & $\left\{\begin{array}{l}\dot{x}=\alpha-y^{2} \\
\dot{y}=-z+x y \\
\dot{z}=y\end{array}\right.$ & $\alpha-y^{2}$ & $y$ & 0 & -1 \\
\hline 7 & Rossler founded system $(x \rightleftarrows y)^{*}$ & $\left\{\begin{array}{l}\dot{x}=a y-y^{2}-b x \\
\dot{y}=z \\
\dot{z}=-x-y\end{array}\right.$ & $a y-y^{2}$ & 0 & -1 & 1 \\
\hline 8 & Rucklidge attractor $(x \rightleftarrows y)^{*}$ & $\left\{\begin{array}{l}\dot{x}=y \\
\dot{y}=\lambda x-x y-x z \\
\dot{z}=x^{2}-z\end{array}\right.$ & $y$ & $\lambda-z$ & $x$ & 0 \\
\hline 9 & Financial system $(x \rightleftarrows y)^{*}$ & $\left\{\begin{array}{l}\dot{x}=1-y^{2}-b x \\
\dot{y}=z+(x-a) y \\
\dot{z}=-y-c z\end{array}\right.$ & $1-y^{2}$ & $y$ & 1 & 0 \\
\hline
\end{tabular}

${ }^{*} x$ and $y$ are replaced with each other, just to adopt the chaotic systems with the general Model (1).

where $V_{t}, V_{x}$, and $V_{x x}$ denote the partial derivative on $t$, the gradient of $V(t)$, and the Hessian matrix of $V(t)$, respectively, and $\operatorname{tr}\{\cdot\}$ is the sum of the main diagonal entries.

Theorem 5. Stochastic chaotic system (3) is asymptotically stable in mean-square sense with

$$
\begin{aligned}
& {\left[\begin{array}{l}
u_{1}(t) \\
u_{2}(t) \\
u_{3}(t)
\end{array}\right]} \\
& =-\left(\left[\begin{array}{c}
f(s(t))+y(t) \cdot g(s(t))+z(t) \cdot h(s(t)) \\
0 \\
0
\end{array}\right]\right. \\
& \left.+\frac{1}{2} G^{T} G\left[\begin{array}{l}
x(t) \\
y(t) \\
z(t)
\end{array}\right]\right) .
\end{aligned}
$$

Proof. For designing control law in Lyapunov's direct approach (without linearization), at first, Lyapunov function is regarded as

$$
V(t)=\frac{1}{2}\left(x^{2}(t)+y^{2}(t)+z^{2}(t)\right) .
$$

Notice that $E\{V(t)\} \geq 0$. Using Itô differential formula given in Definition 4, the stochastic differential of $V(t)$ along the trajectories of the system (3) turns out to be

$$
\begin{aligned}
d V(t)= & L V(t) d t+x(t) \cdot n_{1}(s(t), t) d \omega_{1} \\
& +y(t) \cdot n_{2}(s(t), t) d \omega_{2} \\
& +z(t) \cdot n_{3}(s(t), t) d \omega_{3} .
\end{aligned}
$$

By substituting $V_{t}, V_{x}$, and $V_{x x}$ in (6), $L V(t)$ is rewritten as follows:

$$
\begin{aligned}
L V(t)= & x(t) \cdot f(s(t))-\alpha x^{2}(t) \\
& +x(t) \cdot u_{1}(t)+x(t) y(t) \cdot g(s(t)) \\
& -\beta y^{2}(t)+y(t) \cdot u_{2}(t)+x(t) z(t) \cdot h(s(t)) \\
& -\gamma z^{2}(t)+z(t) \cdot u_{3}(t) \\
& +\frac{1}{2}\left(n_{1}^{2}(s(t), t)+n_{2}^{2}(s(t), t)+n_{3}^{2}(s(t), t)\right) ;
\end{aligned}
$$


in other words, $L V(t)$ is turned to

$L V(t)$

$$
\begin{aligned}
& =\left[\begin{array}{l}
x(t) \\
y(t) \\
z(t)
\end{array}\right]^{T} \\
& \times\left(\left[\begin{array}{c}
f(s(t))-\alpha x(t)+y(t) \cdot g(s(t))+z(t) \cdot h(s(t)) \\
-\beta y(t) \\
-\gamma z(t)
\end{array}\right]\right. \\
& \left.+\left[\begin{array}{l}
u_{1}(t) \\
u_{2}(t) \\
u_{3}(t)
\end{array}\right]\right)+\frac{1}{2}\|N(s(t), t)\|^{2} .
\end{aligned}
$$

Considering Assumption 2, the above equality becomes the below inequality

$$
L V(t)
$$

$$
\begin{aligned}
& \leq\left[\begin{array}{l}
x(t) \\
y(t) \\
z(t)
\end{array}\right]^{T} \\
& \times\left(\left[\begin{array}{c}
f(s(t))-\alpha x(t)+y(t) \cdot g(s(t))+z(t) \cdot h(s(t))] \\
-\beta y(t) \\
-\gamma z(t)
\end{array}\right]\right. \\
& \left.+\left[\begin{array}{l}
u_{1}(t) \\
u_{2}(t) \\
u_{3}(t)
\end{array}\right]\right)+\frac{1}{2}\left\|\left[\begin{array}{l}
x(t) \\
y(t) \\
z(t)
\end{array}\right]\right\|^{2}
\end{aligned}
$$

or can be stated as follows:

$$
\begin{aligned}
& L V(t) \\
& \leq\left[\begin{array}{l}
x(t) \\
y(t) \\
z(t)
\end{array}\right]^{T} \\
& \times\left(\left[\begin{array}{r}
f(s(t))-\alpha x(t)+y(t) \cdot g(s(t))+z(t) \cdot h(s(t))] \\
-\beta y(t) \\
-\gamma z(t)
\end{array}\right]\right. \\
& \left.+\left[\begin{array}{l}
u_{1}(t) \\
u_{2}(t) \\
u_{3}(t)
\end{array}\right]+\frac{1}{2} G^{T} G\left[\begin{array}{l}
x(t) \\
y(t) \\
z(t)
\end{array}\right]\right) .
\end{aligned}
$$

Since $E\{d V(t)\}=E\{L V(t) d t\}$, in order to show that the stochastic chaotic system (3) is asymptotically stable in the mean-square sense, it is enough to have $E\{L V(t) d t\}<0$. Now, let control law be introduced as (7). By substituting proposed control law into (13), it can be obtained that

$$
L V(t) \leq-\left(\alpha x^{2}(t)+\beta y^{2}(t)+\gamma z^{2}(t)\right)
$$

So, by applying input vector (7), stochastic chaotic system (3) is asymptotically stable in mean-square sense.

\section{Simulation Results}

In this section, the performance of proposed control scheme is evaluated by applying the method on two different chaotic systems. The Chen and financial systems are two well-known chaotic systems which are expressed as

$$
\text { Chen system }\left\{\begin{array}{l}
\dot{x}(t)=d_{1} y(t)-y(t) z(t)+c_{1} x(t), \\
\dot{y}(t)=a_{1}(x(t)-y(t)), \\
\dot{z}(t)=x(t) y(t)-b_{1} z(t),
\end{array}\right.
$$

Financial system $\left\{\begin{array}{l}\dot{x}(t)=1-y^{2}(t)-b_{2} x(t), \\ \dot{y}(t)=z(t)+\left(x(t)-a_{2}\right) y(t), \\ \dot{z}(t)=-y(t)-c_{2} z(t),\end{array}\right.$

where $\left(a_{1}, b_{1}, c_{1}, d_{1}\right)=(35,3,28,-7)$ and $\left(a_{2}, b_{2}, c_{2}\right)=(1$, $0.1,1)$.

4.1. Proposed Controller for Stochastic Chen System. In this case, the efficiency of the proposed Lyapunov's direct method scheme is verified by an example of control of the stochastic Chen system. Here, the stochastic Chen system can be rewritten in the form of (3) as follows:

$$
\begin{aligned}
d x= & \left(d_{2} y(t)-y(t) z(t)+c_{2} x(t)+u_{1}(t)\right) d t \\
& +(2 \sin (0.3 \pi x(t))(x(t)+y(t))) d \omega_{1}, \\
d y= & \left(a_{2}(x(t)-y(t))+u_{2}(t)\right) d t+(\sin (0.2 \pi t)(z(t))) d \omega_{2}, \\
d z= & \left(x(t) y(t)-b_{2} z(t)+u_{3}(t)\right) d t \\
& +(2 \cos (0.1 \pi y(t))(y(t)+z(t))) d \omega_{3} .
\end{aligned}
$$

In the absence of the controller, the stochastic Chen system (17) exhibits a chaotic behavior as shown in Figure 1.

For this case, it is easy to see that

$$
\begin{aligned}
n_{1}^{2}(s(t), t) & =(2 \sin (0.3 \pi x(t))(x(t)+y(t)))^{2} \\
& \leq 4\left(x^{2}(t)+y^{2}(t)+2 x(t) y(t)\right), \\
n_{2}^{2}(s(t), t) & =(\sin (0.2 \pi t) z(t))^{2} \leq z^{2}(t), \\
n_{3}^{2}(s(t), t) & =(2 \cos (0.1 \pi y(t))(y(t)+z(t)))^{2} \\
& \leq 4\left(y^{2}(t)+z^{2}(t)+2 y(t) z(t)\right)
\end{aligned}
$$



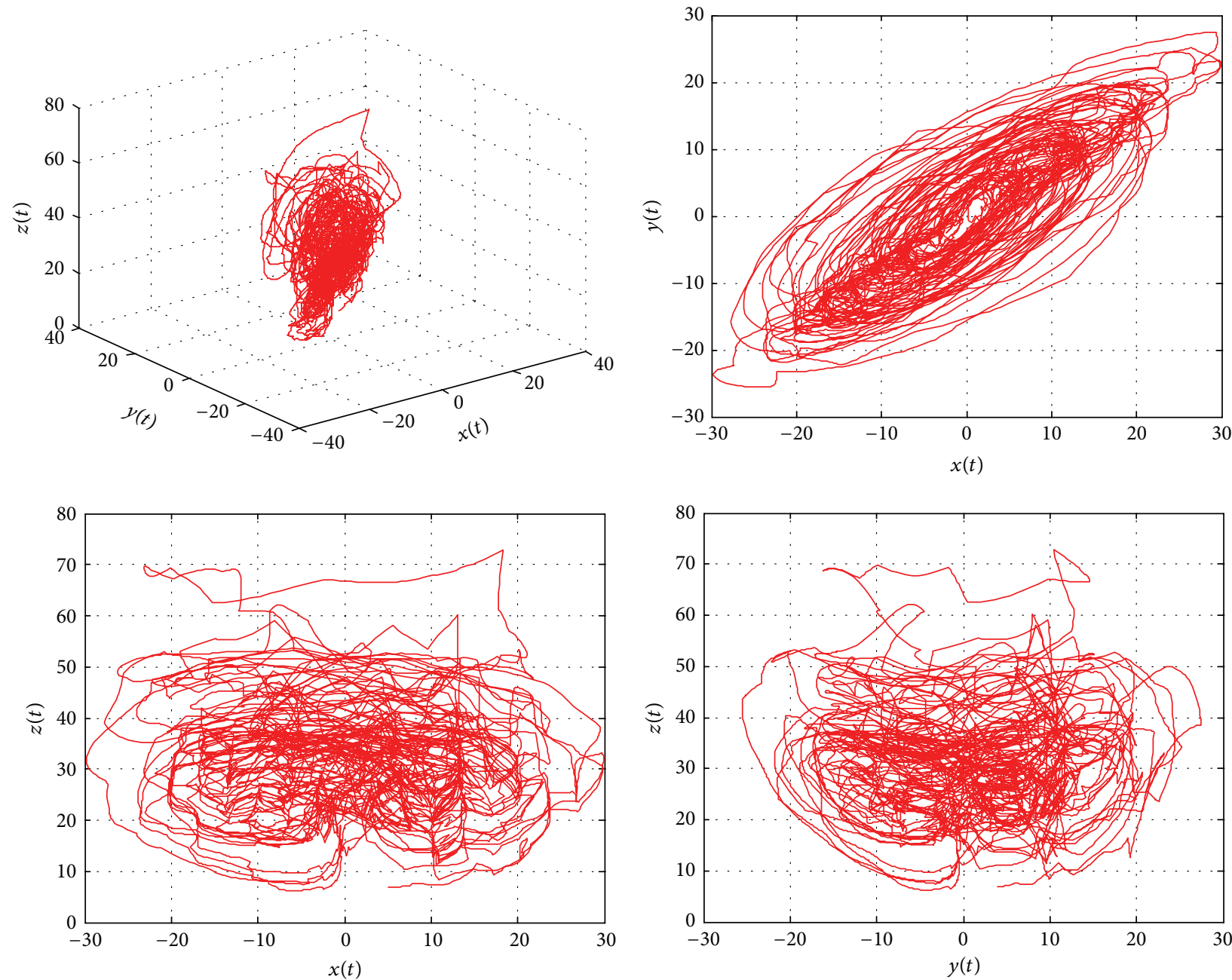

Figure 1: The chaotic trajectories of the stochastic Chen system.

and therefore

$$
\|N(s(t), t)\|^{2} \leq\left[\begin{array}{c}
x(t) \\
y(t) \\
z(t)
\end{array}\right]^{T}\left[\begin{array}{lll}
4 & 4 & 0 \\
4 & 8 & 4 \\
0 & 4 & 5
\end{array}\right]\left[\begin{array}{l}
x(t) \\
y(t) \\
z(t)
\end{array}\right]
$$

According to (7), input signal is acquired as

$$
\left[\begin{array}{l}
u_{1}(t) \\
u_{2}(t) \\
u_{3}(t)
\end{array}\right]=-\left[\begin{array}{c}
(c+2) x(t)+(2+a+d) y(t) \\
2(x(t)+2 y(t)+z(t)) \\
2 y(t)+2.5 z(t)
\end{array}\right]
$$

The simulation results are illustrated with the initial condition

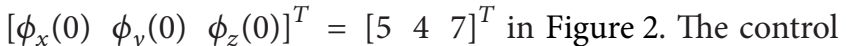
law (20) is applied to the system (17) after five seconds. The left column of Figure 2 illustrates the trajectories of $x(t), y(t), z(t)$ which shows that the obtained theoretic results are feasible and efficient for the controlling stochastic Chen system.
4.2. Proposed Controller for Stochastic Financial System. In this subsection, we will compare our proposed controller with the sliding based controller which is proposed in [17] on the financial system (16). Adding stochastic terms and input vector to the financial system, system (16) can be reformed as

$$
\begin{aligned}
d x= & \left(1-y^{2}(t)-b x(t)+u_{1}(t)\right) d t \\
& +(0.5 \sin (\pi t) x(t)) d \omega_{1}, \\
d y= & \left(z(t)+(x(t)-a) y(t)+u_{2}(t)\right) d t \\
& +(\cos (2 \pi t) y(t)) d \omega_{2}, \\
d z= & \left(-y(t)-c z(t)+u_{3}(t)\right) d t \\
& +(0.25 \cos (t)(z(t)+x(t))) d \omega_{3} .
\end{aligned}
$$

The stochastic system (21) exhibits a chaotic behavior without the controller vector $u(t)$, which is shown in Figure 3. 

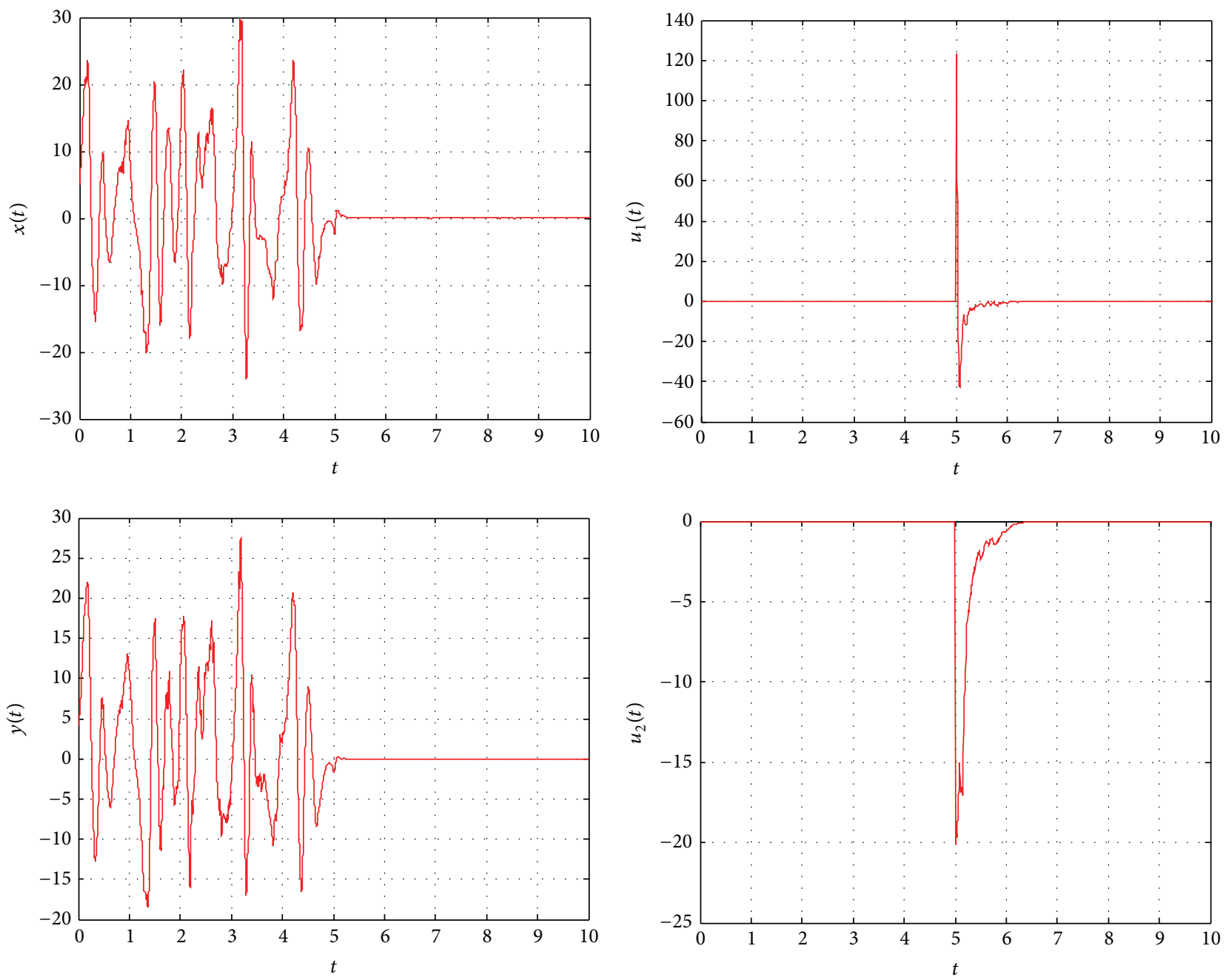

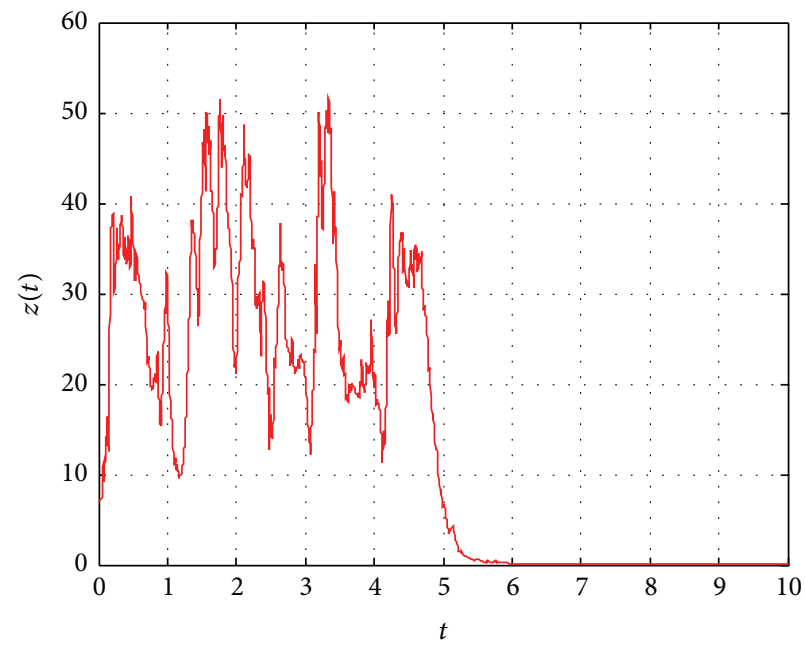

(a)

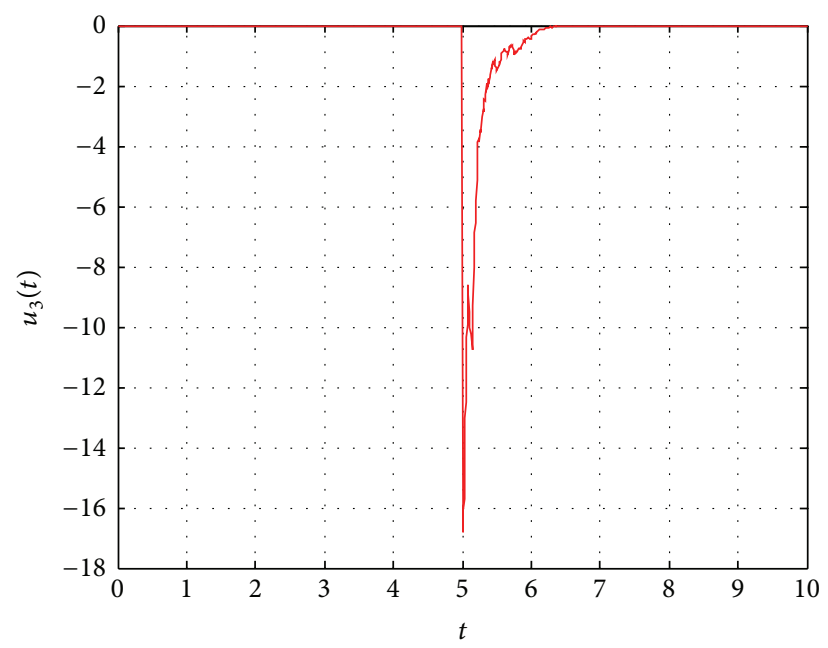

(b)

FIGURE 2: State trajectories of the stabilized stochastic Chen system (a) and input signals (b). 

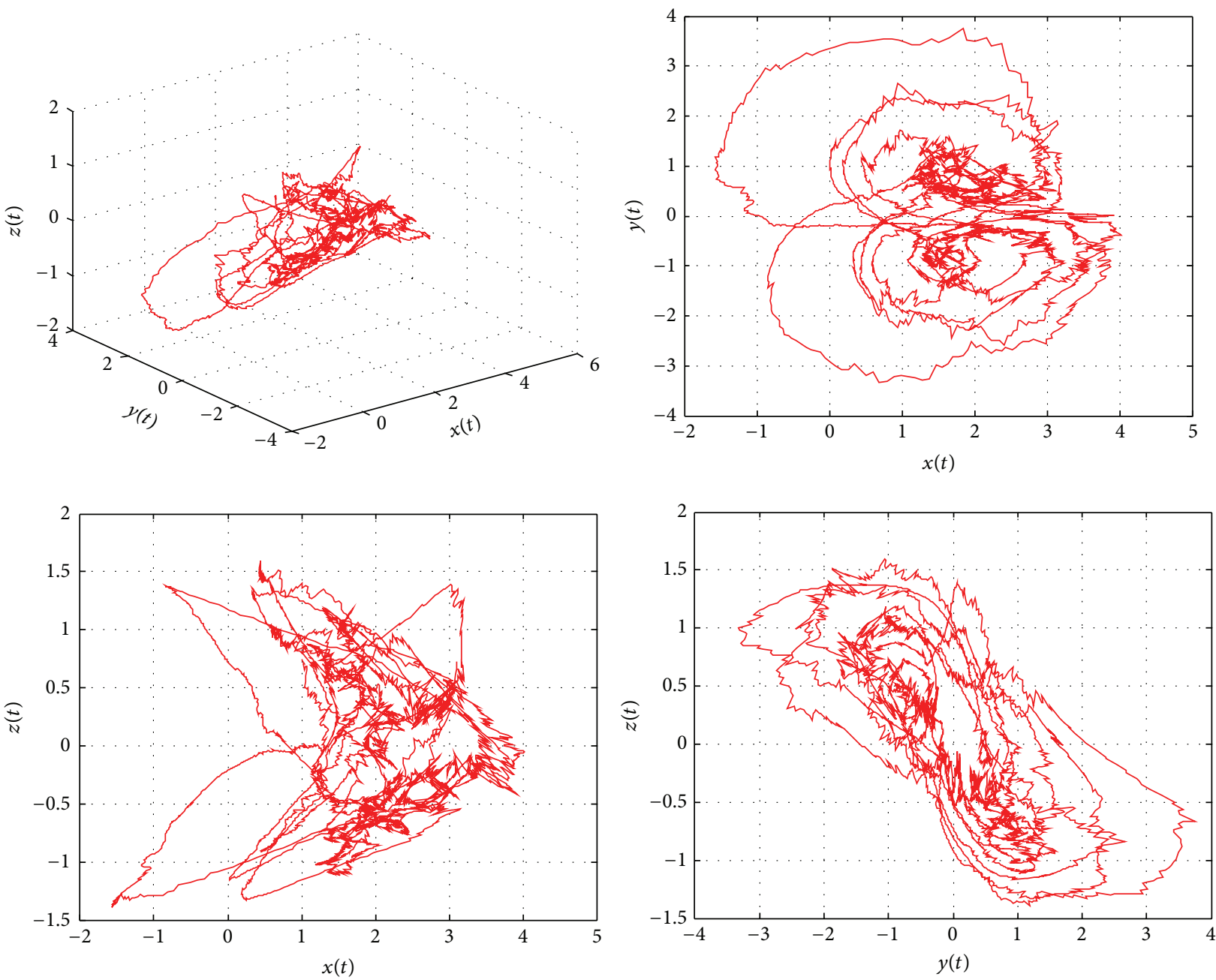

Figure 3: Financial system state space trajectories.

Each $n_{i}(s(t), t), i=1,2,3$, satisfies both locally Lipschitz continuous condition and linear growth condition, and

$$
\begin{aligned}
n_{1}^{2}(s(t), t) & =(0.5 \sin (\pi t) x(t))^{2} \leq 0.25 x^{2}(t), \\
n_{2}^{2}(s(t), t) & =(\cos (2 \pi t) y(t))^{2} \leq y^{2}(t), \\
n_{3}^{2}(s(t), t) & =(0.5 \cos (t)(z(t)+x(t)))^{2} \\
& \leq 0.25\left(x^{2}(t)+z^{2}(t)+2 x(t) z(t)\right) ;
\end{aligned}
$$

therefore,

$$
\|N(s(t), t)\|^{2} \leq\left[\begin{array}{l}
x(t) \\
y(t) \\
z(t)
\end{array}\right]^{T}\left[\begin{array}{ccc}
0.5 & 0 & 0.25 \\
0 & 1 & 0 \\
0.25 & 0 & 0.25
\end{array}\right]\left[\begin{array}{l}
x(t) \\
y(t) \\
z(t)
\end{array}\right] .
$$

Finally, according to (7), our proposed controller is obtained as follows:

$$
\left[\begin{array}{l}
u_{1}(t) \\
u_{2}(t) \\
u_{3}(t)
\end{array}\right]=-\left[\begin{array}{c}
1+0.25 x(t)+0.125 z(t) \\
0.5 y(t) \\
0.125(x(t)+z(t))
\end{array}\right] .
$$

On the other hand, based on sliding mode scheme proposed in [17], input vector is obtained as follows:

$$
\left[\begin{array}{l}
u_{1}^{\prime}(t) \\
u_{2}^{\prime}(t) \\
u_{3}^{\prime}(t)
\end{array}\right]=\left[\begin{array}{c}
-1+y^{2}(t)+b x(t)-0.1 \operatorname{sgn}(x(t)) \\
-z(t)-(x(t)-a) y(t)-0.1 \operatorname{sgn}(y(t)) \\
y(t)+c z(t)-0.1 \operatorname{sgn}(z(t))
\end{array}\right] .
$$

It is worth to mention that, in order to design a sliding mode controller, we have two steps: first constructing a sliding surface and designing an equivalent control law to have a desired system dynamics, second developing a switching control law such that a sliding mode exists on every point of the sliding surface, and thus any states outside the surface are driven to reach the surface in a finite time.

The simulation results are illustrated with the initial condition $\left[\begin{array}{lll}\phi_{x}(0) & \phi_{y}(0) & \phi_{z}(0)\end{array}\right]^{T}=\left[\begin{array}{lll}1 & 0.5 & 0.75\end{array}\right]^{T}$ in Figure 4. Furthermore, the controllers (24) and (25) are applied to the financial system after 25 seconds.

The state $x(t)$ of the system (21) is shown in the left column of Figure 4, in which the first row shows the effect of 

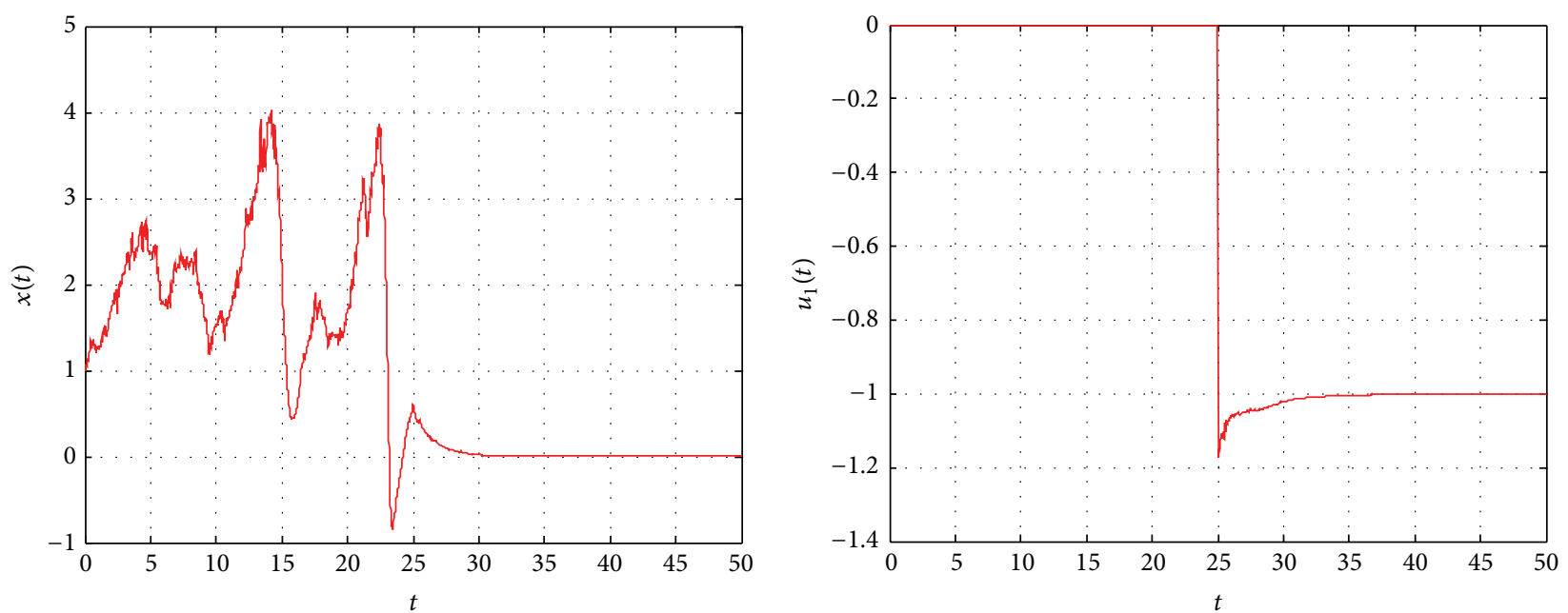

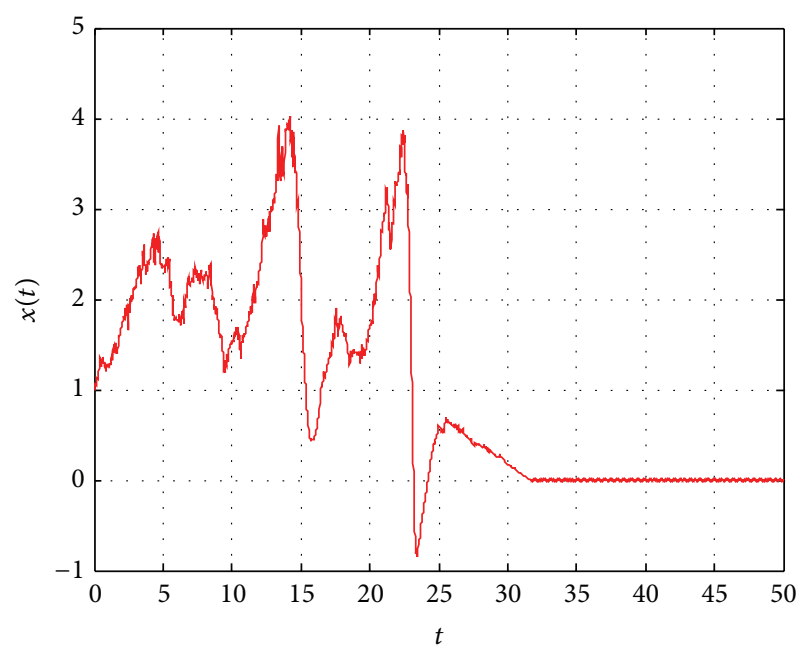

(a)

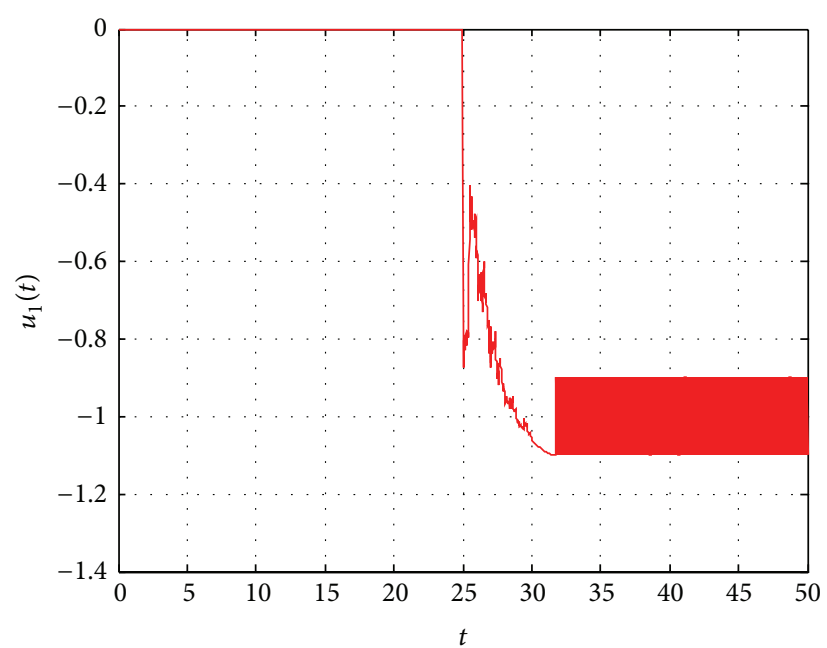

(b)

FIGURE 4: State trajectories of the stabilized stochastic financial system (a) and input signals (b).

our proposed control law and the second row is related to sliding based controller. The states $y(t)$ and $z(t)$ are not shown here due to space limitations. In the right column of Figure 4, the input signals are presented in which the first row is related to Lyapunov's direct method and the second row is related to sliding mode strategy. It should be pointed that, in the last subfigure of Figure 4, the effect of equivalent control law is from 25th second to 32nd second, and next seconds are related to switching control law that causes chattering phenomenon.

It can be seen by using proposed method that not only would the state $x(t)$ converge to zero and the transient response be fast, but also, in comparison with the sliding based input, the input signal is smooth and chattering-free.

\section{Conclusions}

In this paper, the control problem of a group of stochastic chaotic systems is studied. Based on Lyapunov's direct method and Itô formula, the control law has been designed to control chaotic systems. It has been shown that, by applying proposed controller, systems are asymptotically stable in mean square sense and the transient responses are fast. Finally, in order to demonstrate the feasibility and effectiveness, the proposed control scheme is applied to two wellknown chaotic systems.

\section{Conflict of Interests}

The authors declare that there is no conflict of interests regarding the publication of this paper.

\section{References}

[1] J. A. Gallegos, "Nonlinear regulation of a Lorenz system by feedback linearization techniques," Dynamics and Control, vol. 4, no. 3, pp. 277-298, 1994.

[2] A. Razminia and D. F. M. Torres, "Control of a novel chaotic fractional order system using a state feedback technique," Mechatronics, vol. 23, no. 7, pp. 755-763, 2013. 
[3] S. Dadras, H. R. Momeni, and V. J. Majd, "Sliding mode control for uncertain new chaotic dynamical system," Chaos, Solitons and Fractals, vol. 41, no. 4, pp. 1857-1862, 2009.

[4] S. Dadras and H. R. Momeni, "Control of a fractional-order economical system via sliding mode," Physica A: Statistical Mechanics and Its Applications, vol. 389, no. 12, pp. 2434-2442, 2010.

[5] J. Li, W. Li, and Q. Li, "Sliding mode control for uncertain chaotic systems with input nonlinearity," Communications in Nonlinear Science and Numerical Simulation, vol. 17, no. 1, pp. 341-348, 2012.

[6] M. Roopaei, B. R. Sahraei, and T.-C. Lin, "Adaptive sliding mode control in a novel class of chaotic systems," Communications in Nonlinear Science and Numerical Simulation, vol. 15, no. 12, pp. 4158-4170, 2010.

[7] J. Yuan, B. Shi, and W. Ji, "Adaptive sliding mode control of a novel class of fractional chaotic systems," Advances in Mathematical Physics, vol. 2013, Article ID 576709, 13 pages, 2013.

[8] H. Layeghi, M. T. Arjmand, H. Salarieh, and A. Alasty, "Stabilizing periodic orbits of chaotic systems using fuzzy adaptive sliding mode control," Chaos, Solitons and Fractals, vol. 37, no. 4, pp. 1125-1135, 2008.

[9] D. Sadaoui, A. Boukabou, and S. Hadef, "Predictive feedback control and synchronization of hyperchaotic systems," Applied Mathematics and Computation, vol. 247, pp. 235-243, 2014.

[10] C. Wu, Y. Lei, and T. Fang, "Stochastic chaos in a Duffing oscillator and its control," Chaos, Solitons and Fractals, vol. 27, no. 2, pp. 459-469, 2006.

[11] M. Pascual and P. Mazzega, "Quasicycles revisited: apparent sensitivity to initial conditions," Theoretical Population Biology, vol. 64, no. 3, pp. 385-395, 2003.

[12] W. J. Freeman, "A proposed name for aperiodic brain activity: stochastic chaos," Neural Networks, vol. 13, no. 1, pp. 11-13, 2000.

[13] P. R. Patnaik, "The extended Kalman filter as a noise modulator for continuous yeast cultures under monotonic, oscillating and chaotic conditions," Chemical Engineering Journal, vol. 108, no. 1-2, pp. 91-99, 2005.

[14] N. Kikuchi, Y. Liu, and J. Ohtsubo, "Chaos control and noise suppression in external-cavity semiconductor lasers," IEEE Journal of Quantum Electronics, vol. 33, no. 1, pp. 56-65, 1997.

[15] C. Kyrtsou and M. Terraza, "Stochastic chaos or ARCH effects in stock series? A comparative study," International Review of Financial Analysis, vol. 11, no. 4, pp. 407-431, 2002.

[16] B. Oksendal, Stochastic Differential Equations: An Introduction with Applications, Springer, Berlin, Germany, 3rd edition, 1992.

[17] H. Salarieh and A. Alasty, "Control of stochastic chaos using sliding mode method," Journal of Computational and Applied Mathematics, vol. 225, no. 1, pp. 135-145, 2009.

[18] S. A. Poznyak, Advanced Mathematical Tools for Automatic control Engineers, Elsevier, 2009.

[19] X. Mao, Stochastic Differential Equations and Applications, Horwood, Chichester, UK, 1997.

[20] L. Arnold, Stochastic Differential Equations: Theory and Applications, Wiley, New York, NY, USA, 1972.

[21] A. Friedman, Stochastic Differential Equations and Applications, vol. 1, Academic Press, New York, NY, USA, 1975.

[22] S. Xu, P. Shi, F. Chunmei, G. Yiqian, and Z. Yun, "Robust observers for a class of uncertain nonlinear stochastic systems with state delays," Nonlinear Dynamics and Systems Theory, vol. 4, no. 3, pp. 369-380, 2004.
[23] Y. Chen, A. Xue, S. Zhou, and R. Lu, "Delay-dependent robust control for uncertain stochastic time-delay systems," Circuits, Systems, and Signal Processing, vol. 27, no. 4, pp. 447-460, 2008. 

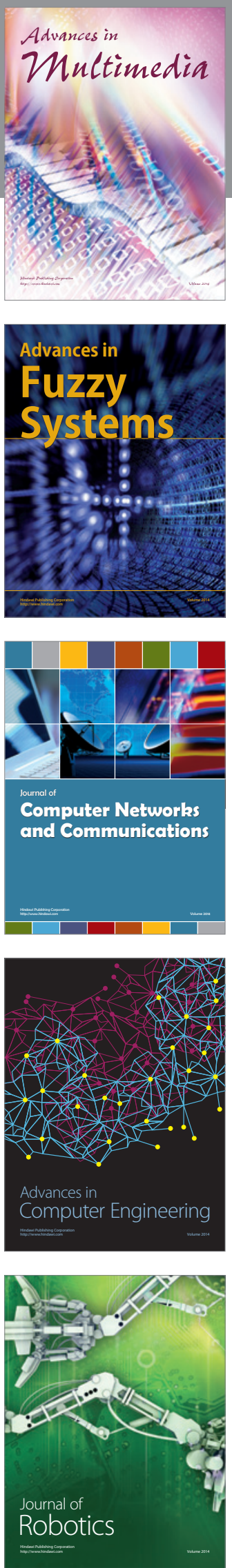

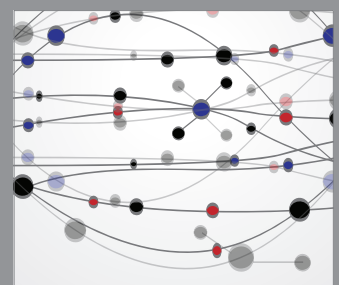

The Scientific World Journal
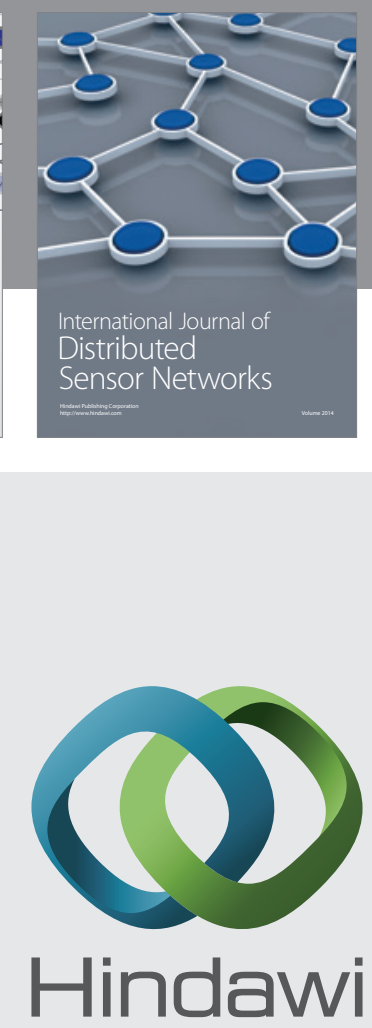

Submit your manuscripts at

http://www.hindawi.com
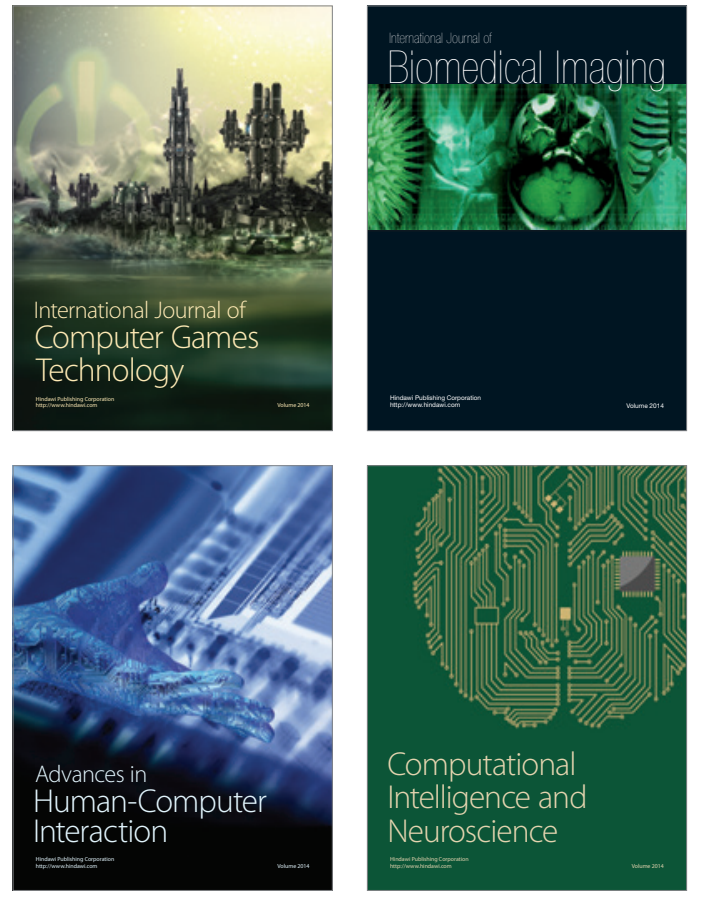
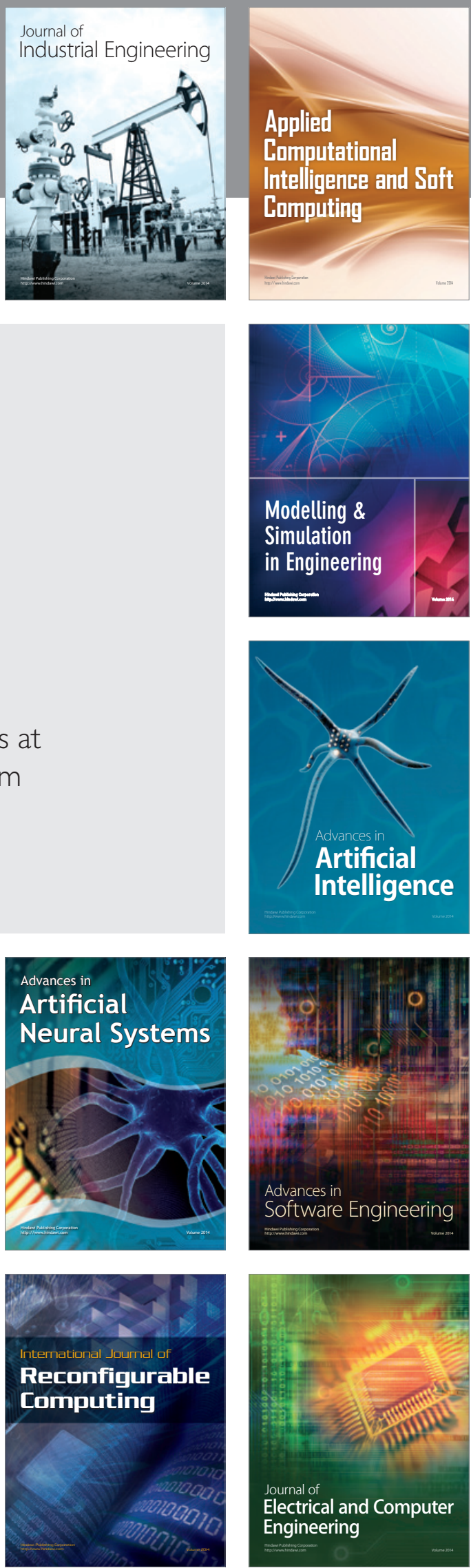